\title{
Magnetic field enhanced convective diffusion of iron oxide nanoparticles in an osmotically disrupted cell culture model of the blood-brain barrier
}

\author{
This article was published in the following Dove Press journal: \\ International Journal of Nanomedicine \\ 20 June 2014 \\ Number of times this article has been viewed
}

\author{
Zhizhi Sun' \\ Matthew Worden ${ }^{2}$ \\ Yaroslav Wroczynskyj ${ }^{3}$ \\ Vinith Yathindranath ${ }^{4}$ \\ Johan van Lierop ${ }^{3}$ \\ Torsten Hegmann 1,2,4,5 \\ Donald W Miller' \\ 'Department of Pharmacology and \\ Therapeutics, University of Manitoba, \\ Winnipeg, Manitoba, Canada; \\ ${ }^{2}$ Department of Chemistry and \\ Biochemistry, Kent State University, \\ Kent, OH, USA; ${ }^{3}$ Department of \\ Physics and Astronomy, ${ }^{4}$ Department \\ of Chemistry, University of Manitoba, \\ Winnipeg, Manitoba, Canada; \\ ${ }^{5}$ Chemical Physics Interdisciplinary \\ Program, Liquid Crystal Institute, \\ Kent State University, Kent, OH, USA
}

Purpose: The present study examines the use of an external magnetic field in combination with the disruption of tight junctions to enhance the permeability of iron oxide nanoparticles (IONPs) across an in vitro model of the blood-brain barrier (BBB). The feasibility of such an approach, termed magnetic field enhanced convective diffusion (MFECD), along with the effect of IONP surface charge on permeability, was examined.

Methods: The effect of magnetic field on the permeability of positively (aminosilane-coated [AmS]-IONPs) and negatively (N-(trimethoxysilylpropyl)ethylenediaminetriacetate [EDT]IONPs) charged IONPs was evaluated in confluent monolayers of mouse brain endothelial cells under normal and osmotically disrupted conditions.

Results: Neither IONP formulation was permeable across an intact cell monolayer. However, when tight junctions were disrupted using D-mannitol, flux of EDT-IONPs across the bEnd.3 monolayers was $28 \%$, increasing to $44 \%$ when a magnetic field was present. In contrast, the permeability of AmS-IONPs after osmotic disruption was less than 5\%. The cellular uptake profile of both IONPs was not altered by the presence of mannitol.

Conclusions: MFECD improved the permeability of EDT-IONPs through the paracellular route. The MFECD approach favors negatively charged IONPs that have low affinity for the brain endothelial cells and high colloidal stability. This suggests that MFECD may improve IONP-based drug delivery to the brain.

Keywords: drug delivery, iron oxide nanoparticles, permeability, blood-brain barrier, magnetic targeting

\section{Introduction}

Central nervous system (CNS) drug candidates have the lowest approval rate compared with other drug categories during the drug development process. ${ }^{1}$ A principle challenge toward developing CNS drugs is obtaining sufficient permeability across the blood-brain barrier (BBB) to achieve adequate therapeutic concentrations in the brain. The brain capillary endothelial layer is morphologically distinct compared with other vascular beds because it lacks fenestrations, has reduced pinocytic activity, and contains complex tight junctions. ${ }^{2}$ In addition to the restricted paracellular diffusion of solutes, the expression of P-glycoprotein, multidrug-resistance protein, breast cancer-resistance protein, and various other drug efflux transporters in brain microvessel endothelial cells limit the transcellular diffusion of a wide variety of solutes and drugs. ${ }^{3-6}$ Although it is generally accepted that large molecules have limited BBB permeability, smaller molecules also face restricted BBB permeability, with 
an estimated $95 \%$ of traditional small molecule compounds in drug discovery libraries unable to penetrate the $\mathrm{BBB}$ in pharmacologically relevant amounts. ${ }^{7}$ Therefore, the effective treatment of CNS diseases requires an understanding of both the pharmacological target and the BBB permeability properties of a drug.

The applications of iron oxide nanoparticles (IONPs) as potential drug carriers have been explored actively over the last few years..$^{8-12}$ To date, the application of IONPs for imaging and therapeutic interventions of CNS disorders remains a great challenge. Under normal conditions, the permeability of IONPs across the BBB is restricted with little to no detectable penetration of IONPs reported with in vitro and in vivo models. ${ }^{13,14}$ Indeed, the majority of studies reporting IONP delivery to the brain have been under conditions in which the BBB is compromised, such as in brain tumor models, where the enhanced permeability and retention effect of the tumor contribute to the accumulation of IONPs. ${ }^{15,16}$ However, as the extent of BBB disruption observed in brain tumor models is variable and is dependent on the stage of tumor development, ${ }^{17}$ other methods of delivery to the brain are required.

An additional concern with nanoparticles is their distribution in nontarget tissue. Nonspecific uptake of IONPs in the body will lead to systemic distribution and potential toxicity. The reticuloendothelial system in the liver, spleen, and circulating macrophages efficiently eliminates the majority of intravenously administered IONPs, preventing delivery to the target tissue site. One way to circumvent this problem is to modify the surface chemistry of the IONPs, as nanoparticlecell interactions depend on the physiochemical properties of the nanoparticles (NPs). Recently, we have established the biocompatibility and cell accumulation of both positively and negatively charged IONPs in a variety of different CNS relevant cell systems. ${ }^{18}$ Although these studies showed a high degree of biocompatibility with all cell types examined, there was a substantial difference in cellular accumulation, with the positively charged IONPs having much greater uptake in brain microvessel endothelial cells compared with the negatively charged IONPs of similar size. ${ }^{18}$

The present study extends these initial observations by evaluating the effect of IONP surface charge on permeability across an in vitro cell culture model of the BBB. It was hypothesized that negatively charged IONPs would favor paracellular diffusion routes, whereas positively charged IONPs would primarily undergo transcellular vesicular transport. Further, it was postulated that although the paracellular route would normally be insufficient to support IONP delivery, transient disruption coupled with an externally applied magnetic field, which we have termed magnetic field enhanced convective diffusion (MFECD), could be used to enhance BBB penetration.

\section{Materials and methods Materials}

All chemical reagents were purchased from Sigma Aldrich (St Louis, MO, USA), and cell culture reagents were purchased from Life Technologies Inc. (Thermo Fisher Scientific, Waltham, MA, USA), unless otherwise specified.

\section{Nanoparticle synthesis and characterization}

Iron oxide nanoparticles were prepared under mild conditions at room temperature, as previously described..$^{19}$ Aminosilane coating (AmS) of the IONPs was performed by rapidly adding 3-aminopropyltriethoxysilane $(17 \mathrm{mmol})$ to the IONPs in a reaction vessel and stirring continuously overnight at room temperature. The crude product was purified by dialysis with molecular weight cut-off 6000-8000 Spectra/ Por 1 dialysis membrane (Spectrum Labs Inc., Rancho Donminguez, CA, USA). The resulting AmS-IONPs had a magnetite $\left(\mathrm{Fe}_{3} \mathrm{O}_{4}\right)$ core and aminosilane outer shell with free amine functional groups. The EDT-IONPs were prepared by adding $\mathrm{N}$-(trimethoxysilylpropyl)ethylenediaminetriacetate trisodium salt (EDT, $3 \mathrm{mmol}$, from a solution concentration of $45 \%$ in water; Gelest, Morrisville, PA, USA) directly to a reaction vessel containing IONPs. The mixture was allowed to react overnight with stirring, and the final product was purified by dialysis, as described for AmS-IONPs. Both NPs were filtered through a $0.2 \mu \mathrm{m}$ nylon filter before experiments.

The IONP size distribution in DI water was determined initially through photon correlation spectroscopy (PCS) at a fixed scattering angle $\left(90^{\circ}\right)$, using a Horiba Nano-Partica SZ-100 series instrument (Horiba Instruments Inc., Irvine, CA, USA). The same instrument allowed for the assessment of particle surface charge (zeta potential) by the measurement of IONP electrophoretic mobilities, using phase analysis light scattering. Further characterization of IONP hydrodynamic size distribution was performed by PCS, using a Photocor Complex (Photocor Instruments Inc., College Park, MD, USA) and allowing for measurements over a range of scattering angles.

\section{Cell culture}

A mouse brain-derived microvessel endothelial cell line, bEnd.3 (American Type Tissue Culture Collection, Manassas, VA, USA), was used as a cell culture model of the BBB. The bEnd. 3 cells (passage number 30-50) were cultured in 
Dulbecco's Modified Eagle's Medium (DMEM; Hyclone, Logan, UT, USA) supplemented with 10\% heat-inactivated fetal bovine serum (Hyclone) and 50 units $/ \mathrm{mL}$ penicillin and streptomycin (MP Biomedicals, Solon, OH, USA) at $37^{\circ} \mathrm{C}$ and $5 \% \mathrm{CO}_{2}$. Cells were expanded in $\mathrm{T}-75$ tissue culture flasks and then passaged and seeded at $2 \times 10^{5}$ cells per well on six well plates and six well inserts for uptake and permeability studies, respectively. Culture medium was changed every 2 days. All experiments were performed on confluent monolayers, typically on day 4 or 5 postseeding.

\section{Cellular uptake of IONP compositions}

Confluent monolayers of bEnd.3 cells grown on six well culture plates (Costar, Lowell, MA, USA) were treated with culture media or culture media with $1.4 \mathrm{M}$ mannitol containing various IONP concentrations $(2.5-50 \mu \mathrm{g} / \mathrm{mL}$ Fe). After treatment with IONPs, the cells were placed in a humidified $\mathrm{CO}_{2}$ incubator maintained at $37^{\circ} \mathrm{C}$. After 5 hours, the culture media was removed and the cell monolayers were washed three times with ice-cold phosphate-buffered saline to remove the unbound NPs. Cells were lysed with $500 \mu \mathrm{L}$ of $0.2 \mathrm{M} \mathrm{NaOH}$, and the IONP content was determined from the Ferrozine assay described previously. ${ }^{18}$ Cellular accumulation was examined in both the presence and absence of a static magnetic field. For studies involving a magnetic field, the cells were placed over a platform containing commercial cylindrical rare-earth magnets (Nd-Fe-B, $19 \mathrm{~mm}$ diameter, $3 \mathrm{~mm}$ height). Under these conditions, the cell monolayer was positioned approximately $1 \mathrm{~mm}$ above the magnets (field strength, $0.13 \mathrm{~T}$ ). Cells with IONPs remained exposed to the magnetic field for the duration of the experiment.

\section{Permeability studies}

Permeability studies were performed on confluent monolayers of bEnd. 3 cells grown on semipermeable polycarbonate (PC) membrane inserts ( $3 \mu \mathrm{m}$ pore size; $24 \mathrm{~mm}$ diameter; Corning Inc., Corning, NY, USA) and maintained with DMEM. Permeability was assessed by adding $1.5 \mathrm{~mL}$ of $50 \mu \mathrm{g} / \mathrm{mL}$ of either AmS-IONPs or EDT-IONPs to the apical (luminal) compartment and incubate at $37^{\circ} \mathrm{C}$ in a $\mathrm{CO}_{2}$ incubator in the presence and absence of a static magnetic field, as described earlier. Under these conditions, the cell monolayer was positioned approximately $3 \mathrm{~mm}$ above the magnets (magnetic field strength, $0.06 \mathrm{~T}$ ). To determine the integrity of the cell monolayers, 70,000 molecular weight fluorescein-labeled dextran (FDX; $250 \mu \mathrm{g} / \mathrm{mL}$; Life Technologiess Inc) was also added to the apical compartment of each monolayer at the beginning of the permeability study, and its appearance in the basolateral compartment was determined using a Biotek Synergy HT plate reader (excitation $488 \mathrm{~nm}$ and emission $510 \mathrm{~nm}$; BioTek Instruments Inc., Winooski, VT, USA). At various times, the IONP concentration in the basolateral compartment was determined by the Ferrozine assay. Quantitative assessments of IONP permeability were determined by the fraction of Fe in the basolateral compartment relative to the amount of Fe loaded in the apical compartment. The apparent permeability coefficients $\mathrm{P}$ for FDX and IONPs were calculated using the following equation:

$$
P=\frac{\Delta Q / \Delta t}{A C_{0}}
$$

Where $\Delta Q / \Delta t$ is the linear appearance rate of the compounds on the receiver, $\mathrm{A}$ is the membrane surface area, and $\mathrm{C}_{0}$ is the initial concentration in the donor chamber.

In separate studies, permeability of the IONP compositions was examined in bEnd.3 monolayers treated with DMEM containing 1.4 M mannitol to disrupt the tight junctions and reduce monolayer integrity.

\section{Analytical assay for measuring IONPs}

Quantitative determination of IONP content in cell and media samples was performed using the Ferrozine assay. ${ }^{18}$ As the Ferrozine assay is an absorbance-based assay for determining ionic iron concentrations, IONPs in the cell lysate and media samples were first solubilized by adding $500 \mu \mathrm{L}$ concentrated $\mathrm{HCl}(\sim 12 \mathrm{M})$ to $500 \mu \mathrm{L}$ cell lysate or media samples. This mixture was incubated for 1 hour at room temperature with gentle shaking and then neutralized with $500 \mu \mathrm{L}$ of $12 \mathrm{M} \mathrm{NaOH}$. On neutralization, $120 \mu \mathrm{L}$ hydroxylamine hydrochloride $(2.8 \mathrm{M})$ in $4 \mathrm{M} \mathrm{HCl}$ was added, and the samples were incubated for 60 minutes at room temperature with gentle shaking. After this incubation, $50 \mu \mathrm{L}$ of $10 \mathrm{M}$ ammonium acetate solution $(\mathrm{pH} 9.5)$ and $300 \mu \mathrm{L}$ of $10 \mathrm{mM}$ ferrozine in $0.1 \mathrm{M}$ ammonium acetate solution was added to each sample. Absorbance was measured at $562 \mathrm{~nm}$, using a Synergy HT plate reader (BioTek). Quantitative assessment of IONP concentration was based on a standard curve prepared using a 1,000 ppm iron atomic absorption standard (Fisher Scientific, Ottawa, ON, Canada). Samples from the cell lysates were normalized for protein content, using a bicinchoninic acid assay protein assay kit (Pierce, Rockford, IL, USA).

\section{Statistical analysis}

All data were expressed as mean \pm standard error of the mean (SEM), and all values were obtained from at least three independent experiments. Statistical significance was evaluated 
using one-way analysis of variance (ANOVA), followed by post hoc comparison of the means using the Fisher's leastsignificant difference test.

\section{Results}

\section{Physicochemical characterization of IONPs}

Characterization of AmS-IONPs by transmission electron microscopy, X-ray diffraction, thermal gravimetric analysis, and Fourier transform infrared spectroscopy has been recently published. ${ }^{21}$ Specific data concerning EDTIONPs can be found in the supplementary information (Figures S1-S4). A schematic drawing of the IONPs used in the present study is provided in Figure 1. The mean hydrodynamic diameters of the AmS-IONPs and EDT-IONPs suspended in water were $25 \pm 1 \mathrm{~nm}$ and $29 \pm 1 \mathrm{~nm}$, respectively, as determined by PCS at a fixed scattering angle (Figure 1). Although the sizes of the IONPs were similar, surface charge characteristics were substantially different, with the AmSIONP having a positive zeta potential of $21 \pm 4 \mathrm{mV}$ compared with $-39 \pm 3 \mathrm{mV}$ observed with the EDT-IONP composition. As the AmS- and EDT-coated IONPs have effectively the same hydrodynamic size in DI water, the selected coatings provided a platform for analyzing the influence of surface charge in uptake and permeability in bEnd. 3 monolayers.

\section{Permeability studies of AmS-IONPs and EDT-IONPs}

Permeability of IONPs across an intact model of the BBB was assessed using confluent monolayers of mouse bEnd.3 cells grown on PC membrane inserts ( $3 \mu \mathrm{m}$ pore size) in the presence and absence of an external magnetic field. In the absence of IONPs, $2 \%$ and $5 \%$ of fluorescein-labeled dextran (FDX-70,000) crossed confluent bEnd.3 monolayers at 8 - and 24-hour times, respectively (Figure $2 \mathrm{~A}$ and $\mathrm{C}$ ). The resulting permeability coefficients for FDX-70,000 in an intact bEnd.3 cells monolayer (Table 1) are consistent with literature reports. ${ }^{22}$ Treatment of the monolayers with either AmS-IONPs or EDT-IONPs $(50 \mu \mathrm{g} / \mathrm{mL})$ had no effect on the magnitude of FDX-70,000 permeability, suggesting that neither of the nanoparticle preparations compromised monolayer integrity (Figure 2A and C; Table 1). In the intact cell monolayers, the permeability of AmS-IONPs was minimal, with less than $1 \%$ of the AmS-IONPs in the basolateral compartment after 24 hours (Figure 2B). The presence of a magnetic field did not enhance the permeability of the AmS-IONPs in the intact bEnd. 3 monolayers. A similar low-permeability profile was observed with EDT-IONPs in either the presence or absence of an external magnetic field (Figure 2D).

Osmotic disruption of the BBB has been extensively studied and is a clinically proven method for enhancing the brain delivery of poorly permeable compounds. As expected, treatment of the cells with $1.4 \mathrm{M}$ mannitol significantly enhanced FDX-70,000 permeability compared with the intact BBB model (Figure 3A and C). However, it should be noted that the apparent permeability coefficients of FDX and both IONP formulations were higher in blank membrane than the disrupted BBB model, suggesting that some cellular resistance to passage of solutes remained even in a disrupted state (Table 1). Under high osmotic conditions, permeability of the positively charged AmS-IONPs was found to be less than 5\% in the absence of a magnetic field, and no significant change was observed with the application of a magnetic field (Figure 3B). This was in contrast to the negatively charged EDT-IONPs that displayed a $30 \%$ flux after 24 hours (Figure 3D) in the mannitol treatment group. In addition, the application of an external magnetic field further enhanced the permeability of EDT-IONPs in the mannitol-treated monolayers, which significantly exceeded the flux observed with the FDX permeability marker at the 24 -hour point (Figure $3 \mathrm{C}$
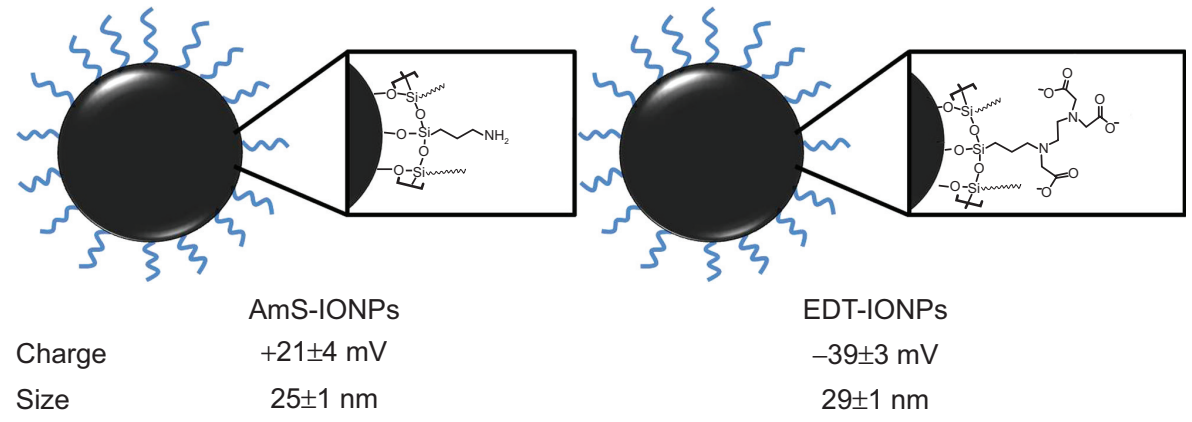

Figure I Schematic drawing of the positively charged AmS-IONPs and negatively charged EDT-IONPs and their physical properties.

Notes: Measurements were performed in triplicate samples, using a Nanopartica SZ-I00 Series Instrument from Horiba (Horiba Instruments Inc., Irvine, CA, USA). Values represent the mean \pm standard error of the mean $(n=3)$.

Abbreviations: AmS-IONPs, aminosilane-coated iron oxide nanoparticles; EDT-IONPs, N-(trimethoxysilylpropyl)ethylenediaminetriacetate-coated iron oxide nanoparticles. 
A

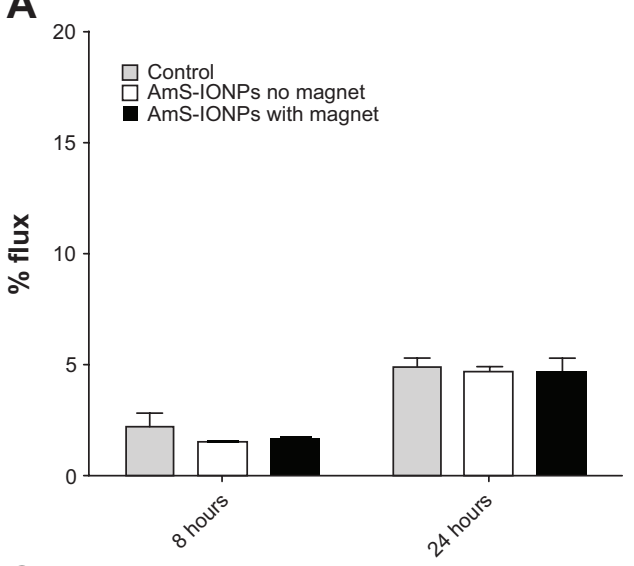

C

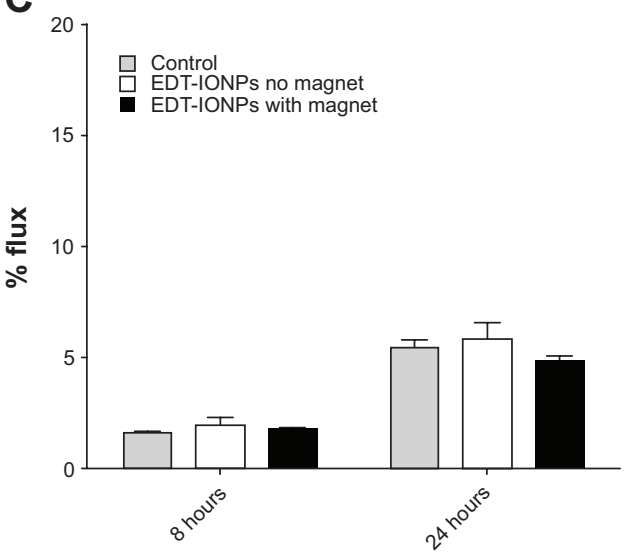

B

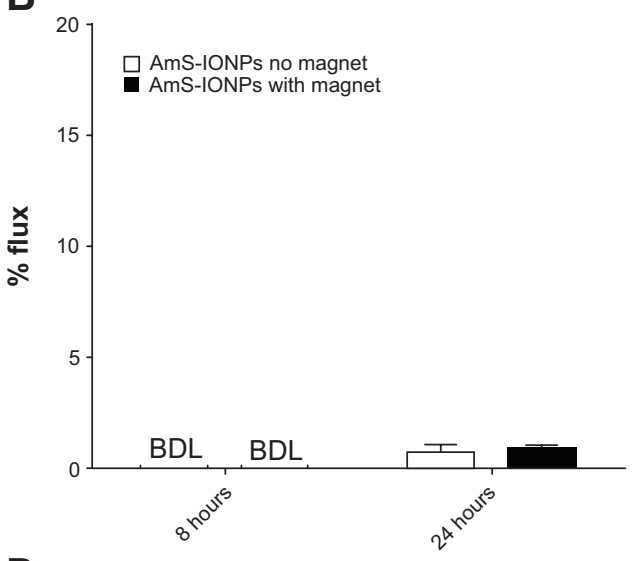

D

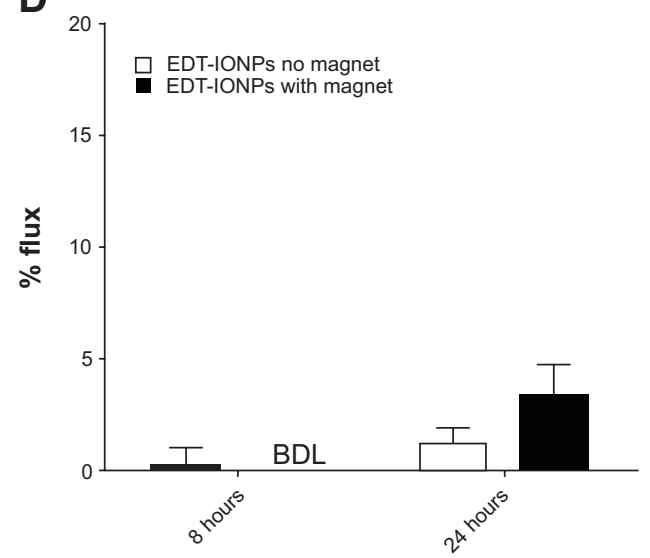

Figure 2 Permeability of FDX-70,000 and IONPs in confluent bEnd.3 cell monolayers in the presence and absence of a magnetic field. FDX-70,000 flux was determined in control monolayers and monolayers treated with AmS-IONPs (A) and EDT-IONPs (C). The permeability of AmS-IONPs (B) and EDT-IONPs (D) was determined at 8 and 24 hours.

Notes: Quantitative determination of IONP permeability was based on the amount of Fe in a basolateral compartment divided by the amount of Fe loaded in an apical compartment and recorded as a percentage. Values represent the mean \pm standard error of the mean of three samples per treatment group.

Abbreviations: AmS-IONPs, aminosilane-coated iron oxide nanoparticles; EDT-IONPs, N-(trimethoxysilylpropyl)ethylenediaminetriacetate-coated iron oxide nanoparticles; FDX, fluorescein-labeled dextran; BDL, below detection limit.

and D). These results support our conclusion that MFECD may improve IONP-based drug delivery to the brain.

To examine potential factors that could be responsible for the limited AmS-IONP permeability in the osmotically disrupted BBB model, the permeability of the IONP formulations was determined across blank PC membranes
(Figure 4A). Both IONPs showed similar trends, with rapid flux to the basolateral compartment observed within 4 hours of incubation. The AmS-IONPs reached 10\% flux at 4 hours and a steady-state permeability of $19 \%$ within 24 hours compared with less than 5\% permeability in the disrupted cell culture model (Figure 4B). This suggests that

Table I Permeability coefficient obtained in blank membrane, intact, and disrupted BBB model for paracellular marker FDX-70,000 MWT, AmS-IONPs, and EDT-IONPs with or without magnet

\begin{tabular}{llll}
\hline & Blank membrane & Intact BBB & Disrupted BBB \\
\hline FDX-70,000 no magnet & $11.3 \pm 0.1$ & $0.20 \pm 0.02$ & $1.25 \pm 0.04$ \\
FDX-70,000 with magnet & $\mathrm{N} / \mathrm{A}$ & $0.18 \pm 0.02$ & $1.1 \mathrm{I} \pm 0.03$ \\
AmS-IONPs no magnet & $2.4 \pm 0.2$ & $0.03 \pm 0.01 * *$ & $0.10 \pm 0.04^{* * *}$ \\
AmS-IONPs with magnet & $\mathrm{N} / \mathrm{A}$ & $0.03 \pm 0.0 I^{* *}$ & $0.1 \mathrm{I} \pm 0.02^{* * *}$ \\
EDT-IONPs no magnet & $\mathrm{II} .1 \pm 0.3$ & $0.04 \pm 0.02^{* *}$ & $1.03 \pm 0.05$ \\
EDT-IONPs with magnet & $\mathrm{N} / \mathrm{A}$ & $0.12 \pm 0.04$ & $1.62 \pm 0.15^{* * * \dagger}$ \\
\hline
\end{tabular}

Notes: The unit is $10^{-6} \mathrm{~cm} /$ second. Values represent the mean $\pm \mathrm{SEM}$ of three samples per treatment group. $* * P<0.0 \mathrm{I}$ compared with FDX-70,000; $* * * P<0.00 \mathrm{I}$ compared with FDX-70,000; ${ }^{\dagger} P<0.001$ compared with same group in the absence of a magnetic field.

Abbreviations: AmS, aminosilane; EDT, N-(trimethoxysilylpropyl)ethylenediaminetriacetate; FDX, fluorescein-labeled dextran; IONPs, iron oxide nanoparticles; SEM, standard error of the mean; BBB, blood-brain barrier; MWT, molecular weight. 

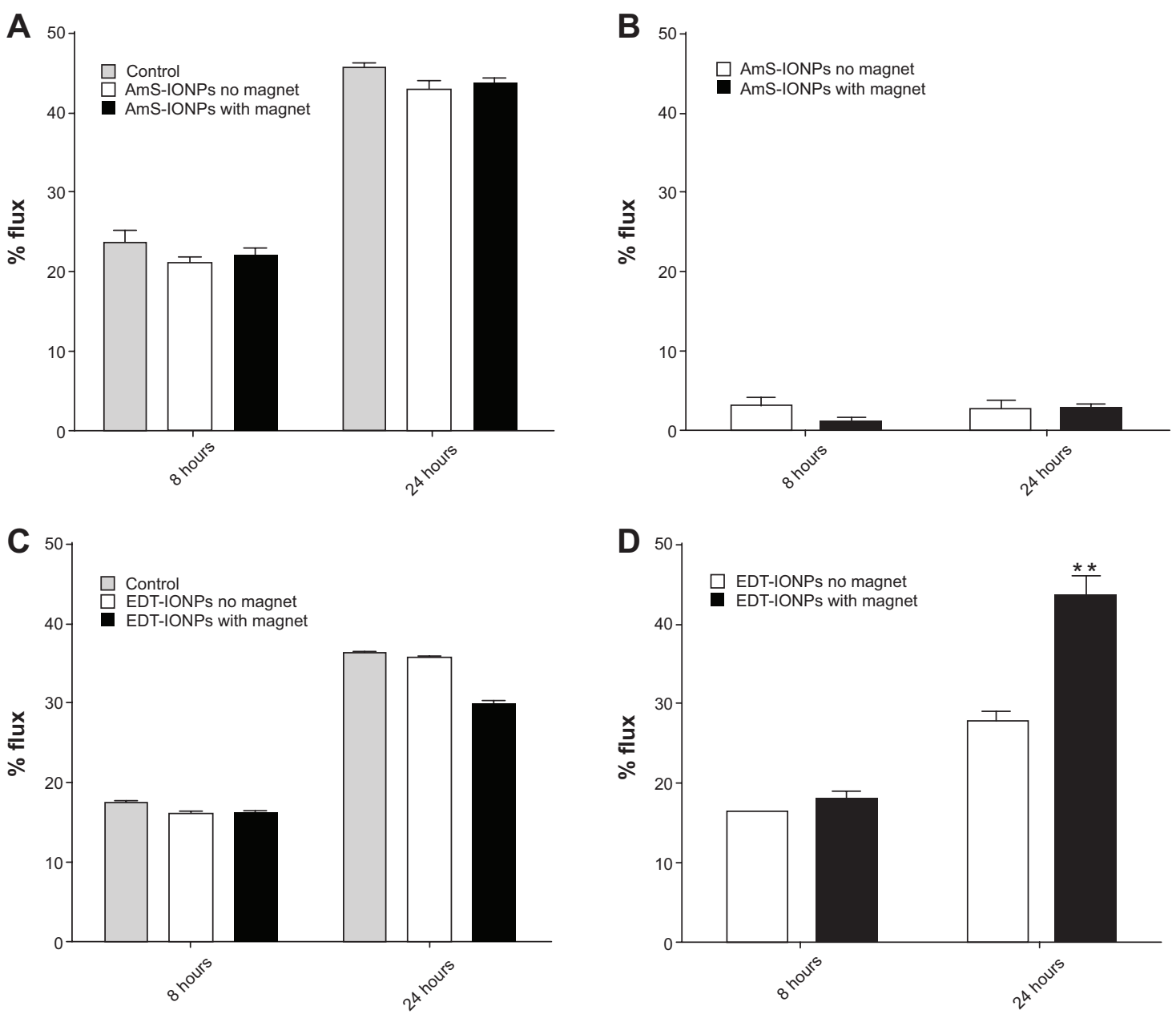

Figure 3 Permeability of fluorescein-labeled dextran-70,000 and IONPs across osmotically disrupted bEnd.3 cell monolayers in the presence and absence of a magnetic field. Fluorescein-labeled dextran-70,000 flux was determined in control monolayers and monolayers treated with AmS-IONPs (A) and EDT-IONPs (C). The permeability of AmS-IONPs (B) and EDT-IONPs (D) was determined at 8 and 24 hours.

Notes: Quantitative determination of IONP permeability was based on the amount of Fe in a basolateral compartment over the amount of Fe loaded in an apical compartment expressed as a percentage. Values represent the mean \pm standard error of the mean of three samples per treatment group. $* * P<0.0 \mathrm{I}$ compared with the same treatment group but without magnetic field.

Abbreviations: AmS-IONPs, aminosilane-coated iron oxide nanoparticles; EDT-IONPs, N-(trimethoxysilylpropyl)ethylenediaminetriacetate-coated iron oxide nanoparticles; IONPs, iron oxide nanoparticles.

the bEnd. 3 cells, regardless of monolayer integrity, act to reduce the permeability of the AmS-IONPs. In contrast, the EDT-IONPs reached $30 \%$ flux at 4 hours and continued to increase to $47 \%$ flux after 24 hours across the blank PC membranes. Compared with an osmotically disrupted model of the BBB, no significant difference was found in the EDTIONP permeability (Figure 4B), indicating the cells were not a limiting factor to EDT-IONP penetration in the disrupted model of the BBB.

\section{IONPs size distribution at physiological $\mathrm{pH}$}

To further understand the reduced permeability of the AmS-IONPs across the blank PC membrane, particle size distributions for the AmS- and EDT-coated IONPs were determined by PCS in water $(\mathrm{pH} 7.8$, as measured before particles were dispersed), with the addition of $\mathrm{NaOH}$ to simulate more closely the $\mathrm{pH}$ conditions used in the permeability studies. Autocorrelation functions of the AmS- and EDT-IONP suspensions were measured at different times after sample preparation (Figure 5) and showed clearly the formation of AmS-IONP aggregates over time. EDT-IONPs did not show aggregation with time, consistent with a stable suspension. The average aggregate diameter of AmS-IONPs was $300 \mathrm{~nm}$ and was observed to vary with scattering angle, indicating nonspherical aggregates. ${ }^{23}$ The fraction of the IONP population displaying aggregation was determined from the relative contributions to the scattered light intensity and was observed to increase during the first few hours after sample preparation, after which it remained constant (Figure 6). Similar results were also found in DMEM cell culture media. As PCS measures only particles in suspension, it is likely that the aggregates grew in size until thermal 
A

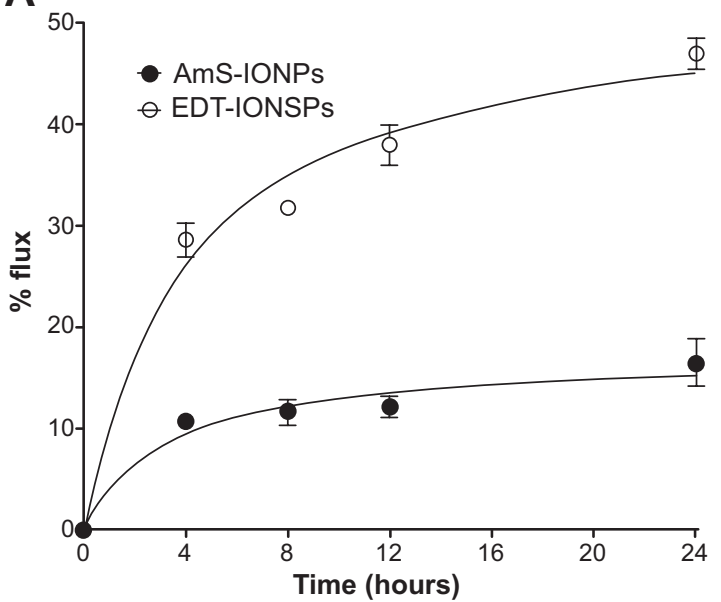

B

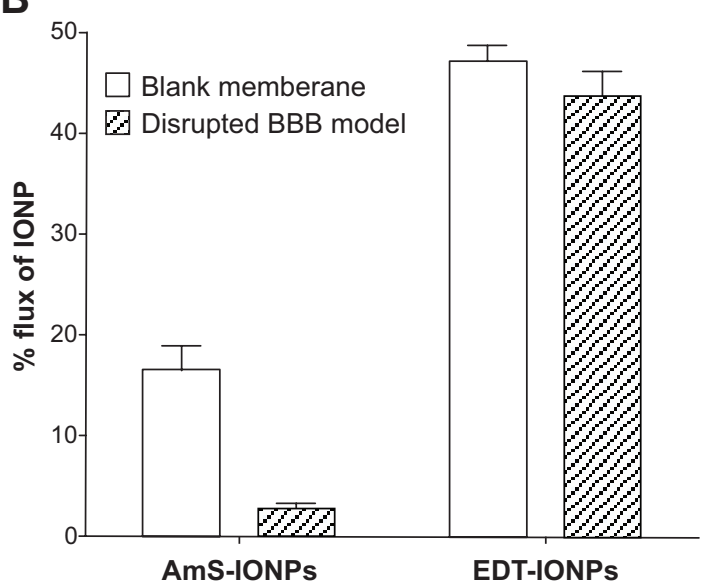

Figure 4 Permeability of aminosilane-coated-iron oxide nanoparticles and $\mathrm{N}$-(trimethoxysilylpropyl)ethylenediaminetriacetate-iron oxide nanoparticles across blank membrane inserts (A) and compared with nanoparticle permeability in a disrupted model of the blood-brain barrier (B).

Note: Values represent the mean \pm standard error of the mean of three samples per treatment group.

Abbreviations: AmS-IONPs, aminosilane-coated iron oxide nanoparticles; EDT-IONPs, N-(trimethoxysilylpropyl)ethylenediaminetriacetate-coated iron oxide nanoparticles; IONP, iron oxide nanoparticle; BBB, blood-brain barrier.

fluctuations were insufficient to keep the aggregates in suspension. A collection of several aggregates with average sizes of $300 \mathrm{~nm}$ could obstruct the $3 \mu \mathrm{m}$ pores of the PC membrane, leading to the observed reduced permeability of the AmS-IONPs in blank membranes.

\section{Cellular uptake of AmS-IONPs and EDT-IONPs in bEnd. 3 monolayers}

To investigate whether the endocytic transport of IONPs was altered by hyperosmotic conditions, the cellular accumulation of both IONPs in bEnd. 3 monolayers was examined (Figure 7). The uptake of AmS-IONPs was found to be concentration-dependent, with a maximum of $30 \mathrm{ng}$ Fe accumulation per microgram protein observed with the $50 \mu \mathrm{g} / \mathrm{mL}$ concentration of AmS-IONPs. Application of a magnetic field enhanced the accumulation of AmS-IONPs in the bEnd. 3 cells by five-fold at all concentrations tested (Figure 7). Similar studies performed with the EDT-IONPs resulted in substantially lower magnitude of cellular uptake compared with AmS-IONPs (Figure 7). In contrast to the AmS-IONPs, application of a magnetic field did not enhance the cell accumulation of EDT-IONPs (Figure 7). Furthermore, hyperosmotic media had no significant effect on the cellular accumulation of either the AmS-IONP or the EDT-IONP formulation (Figure 7).

\section{Discussion}

The present studies describe the potential application of MFECD as an approach for increasing IONPs delivery to the brain. Although NP-based drug delivery is a rapidly progressing field, drug delivery applications in the CNS are severely limited because of the inability of most NPs to cross the BBB. Indeed, few publications have demonstrated transport of the NPs across the BBB. ${ }^{24-26}$ It has been shown that uncoated, oleic acid-coated, and polyvinylamine-coated IONPs were impermeable across an endothelial cell culture model of the BBB, even in the presence of a magnetic field. ${ }^{13}$ In contrast, a size-dependent permeation of polyethylene glycol functionalized (nonmagnetic) gold NPs was reported, using a rat brain microvessel endothelial cell culture model. ${ }^{27}$ However, as these previous studies did not examine monolayer integrity with a permeability marker, the permeability observed may be attributable to the intrinsic leakage of the modeling system used.

This study used the magnetic properties of the IONPs to improve permeability through an MFECD process. With this technique, a magnetic field is used to enhance the bulk flow movement of IONPs. Although there was a tendency for increased permeability of the EDT-IONPs across intact bEnd. 3 monolayers in the presence of a magnetic field, such effects were not statistically significant with the magnetic field strengths achieved in the present study. This was expected, as tight junctions between the endothelial cells would restrict the bulk flow movement of the IONPs. However, enhancement of IONP permeability with MFECD was observed in the bEnd.3 monolayers after osmotic disruption of the tight junctions, using 1.4 M mannitol. The extent of EDT-IONP permeability with MFECD after hyperosmotic disruption of the tight junctions surpassed that of the FDX-70,000 permeability marker, which has roughly the same size as albumin. 

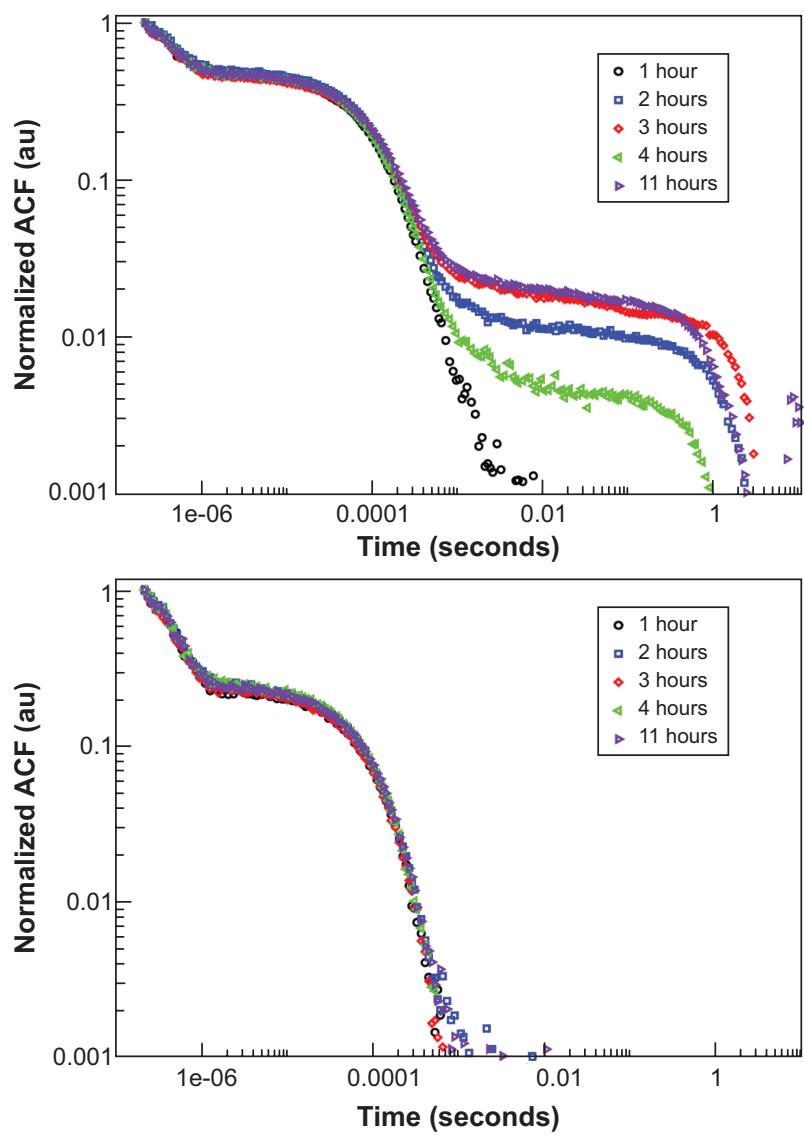

Figure 5 ACFs obtained by photon correlation spectroscopy for AmS-IONPs (top) and EDT-IONPs (bottom) in water at a scattering angle of 90 degrees.

Notes: Symbols indicate time of measurement after suspension. Correlation at larger time scales is indicative of the presence of second, aggregated size distribution, as seen only in the AmS-IONPs.

Abbreviations: ACF, autocorrelation function; AmS-IONPs, aminosilane-coated iron oxide nanoparticles; EDT-IONPs, N-(trimethoxysilylpropyl)ethylenediaminetriacetatecoated iron oxide nanoparticles; PCS, photo correlation spectroscopy.

Transient disruption of the BBB has been used to enhance chemotherapy delivery in both preclinical and clinical treatment of brain tumors. ${ }^{28}$ Several disruption methods have been developed. These include pharmacological disruption via the administration of compounds such as cadherin peptides ${ }^{29}$ and phospholipids, ${ }^{30}$ infusion of hyperosmotic agents such as mannitol, ${ }^{31}$ and physical disruption via the application of techniques such as ultrasound. ${ }^{32}$ Transient opening of the BBB with either osmotic disruption ${ }^{33}$ or focused ultrasound ${ }^{34}$ has been used previously to increase IONP deposition in the brain. The development of focused ultrasound has attracted much attention as a noninvasive method for BBB disruption. When combined with microbubbles, focused ultrasound causes localized and reversible disruption of the BBB. ${ }^{35}$ Although the current study used osmotic disruption to remove water from the cell and break the tight junction complexes, MFECD as an approach to increase delivery of IONPs to the brain would work with any transient disruption technique. The key factors to consider in choosing a disruption proto-
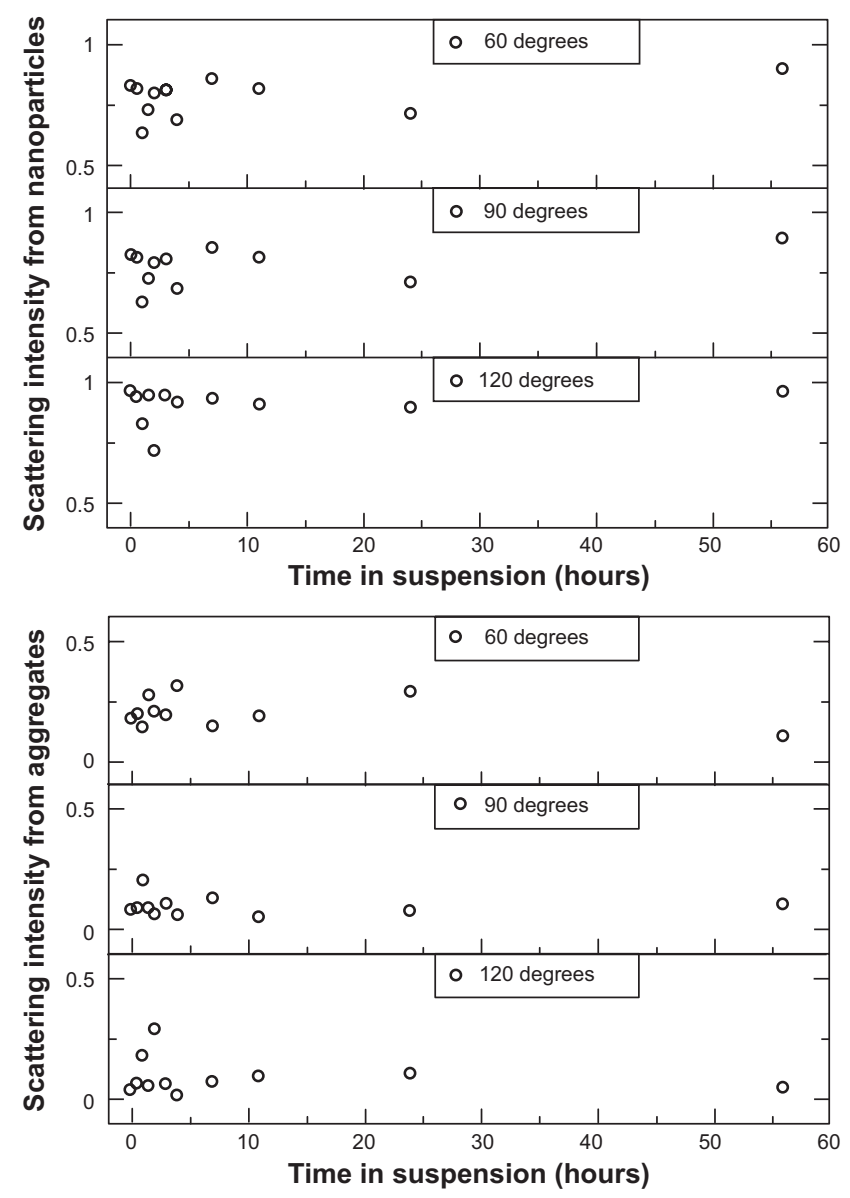

Figure 6 Contributions to the scattered light intensity from AmS-coated nanoparticles (top) and nanoparticle aggregate population (bottom) in water with physiological $\mathrm{pH}$ as a function of time from sample preparation.

Notes: Scattering intensities are reported as the ratio of scattered light from a particular population to the total scattered light intensity. An increase in the relative population of aggregates is observed at small times ( $<3$ hours). The aggregates continue to grow in size past the point of stability and fall out of suspension, no longer contributing to the scattered light intensity.

Abbreviation: AmS, aminosilane.

col are the magnitude and duration of the BBB opening, as prolonged disruption of the $\mathrm{BBB}$ can result in pathological conditions such as extravasation of plasma components, edema formation, and neuroinflammation. The present studies provide the initial proof of concept for MFECD as a means to increase IONP delivery to the brain.

Although the present studies demonstrate the utility of MFECD when combined with disruption of the tight junctions, it is important to note that not all IONP formulations benefit equally from MFECD. The IONPs used in the present study possess the same core structure and size, with surface chemistry modifications leading to different charges. As such, the two IONP formulations examined provide an excellent platform from which to examine the effect of surface charge on MFECD of IONPs across the BBB. Our results confirmed that differently charged IONPs do not pass across the intact endothelial cell layer; however, in the osmotic disrupted 

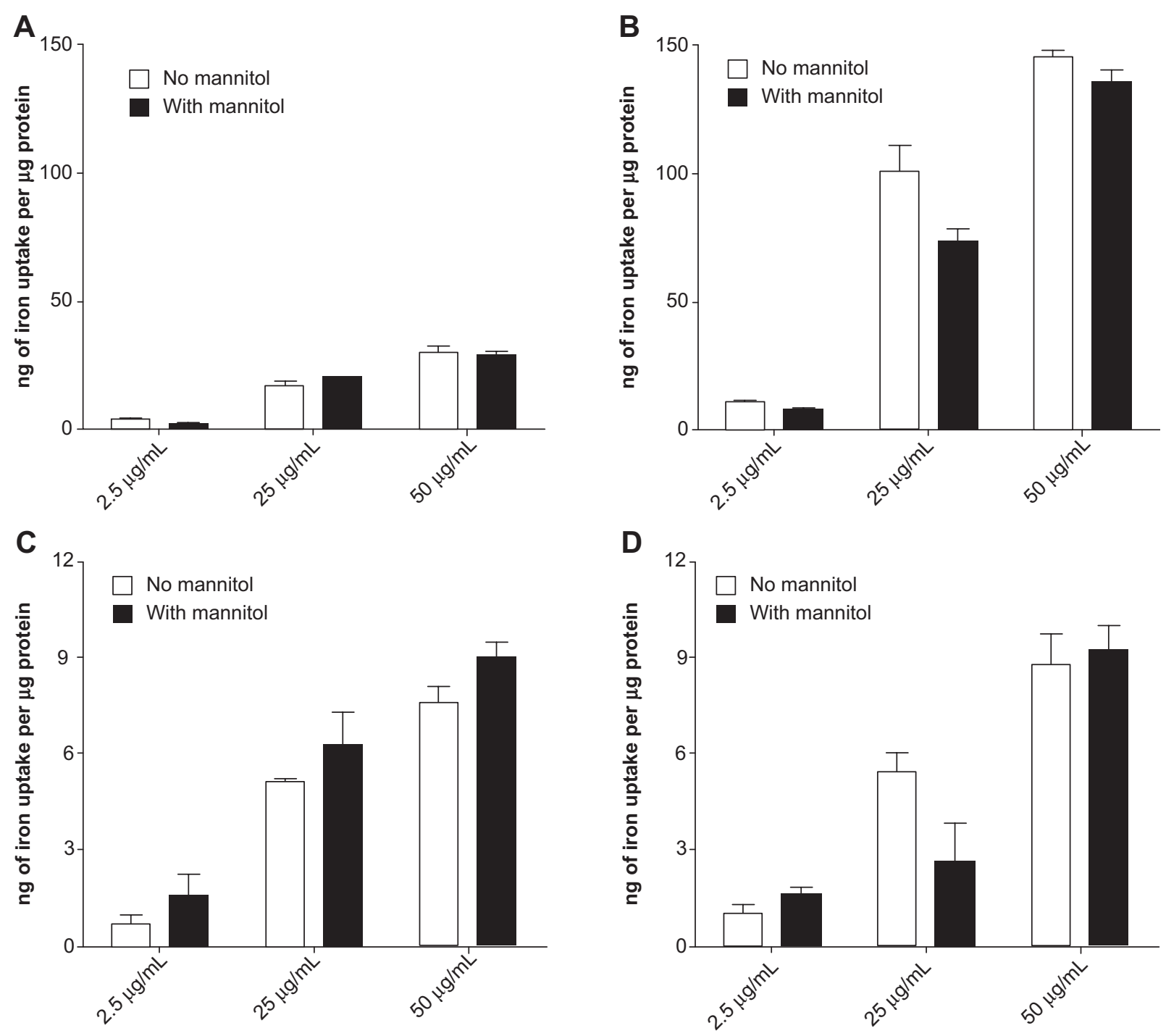

Figure 7 Cell accumulation of AmS-IONPs in the absence $(\mathbf{A})$ and presence $(\mathbf{B})$ of a magnetic field as well as EDT-IONPs uptake in the absence (C) and presence (D) of a magnetic field.

Notes: Values represent the mean \pm standard error of the mean of three samples per treatment group. No significant difference was found between the $D$-mannitol treated and the control group.

Abbreviations: AmS-IONPs, aminosilane-coated iron oxide nanoparticles; EDT-IONPs, N-(trimethoxysilylpropyl)ethylenediaminetriacetate-coated iron oxide nanoparticles.

model of the BBB, the permeability of EDT-IONPs increased substantially and was further enhanced by the presence of a magnetic field. The observed permeability with the EDTIONPs in the absence of a magnetic field was comparable to that of the FDX permeability marker. After a 24-hour exposure to a static magnetic field, the EDT-IONP permeability (44\%) was substantially greater than that observed with FDX (30\%) and was essentially of similar magnitude to that observed in blank membranes without cells. In contrast, AmS-IONPs displayed much lower permeability (less than 5\%) in the osmotic disrupted model of the BBB compared with blank membrane permeability (19\%). This suggests that even in its disrupted state, the bEnd. 3 cells remain a barrier to positively charged AmS-IONPs. The effect of MFECD was most apparent after 24 hours, with the EDT-IONPs having significantly greater permeability than the FDX permeability marker. Although this study examined the effects of MFECD after a relatively long duration of barrier disruption, it is expected that increasing the magnetic field would allow for MFECD under less-extensive BBB disruption conditions. Indeed, studies examining the influence of magnetic field strength are currently ongoing.

In vivo application of an external magnetic field for targeting of IONPs to the brain is certainly more difficult than the current proof-of-concept studies in vitro. Preclinical studies in mice have used Nd-Fe-B magnets placed either on the outside skull or implanted intracranially to apply a local magnetic field. ${ }^{36,37}$ These studies show a greater accumulation of the IONPs in the cortex near the magnet, with progressively lower accumulation in brain tissue farther from the magnetic field. Another group implanted tumor between 
the poles of an electromagnet ${ }^{38,39}$ and demonstrated at least a five-fold increase in magnetic targeting efficiency compared to non-magnetic targeted ones. ${ }^{40}$ Although this is the current state of the art, and has some limitations, it is not out of the question to consider the use of devices for generating "on/off" magnetic fields in discrete brain regions. Such a system would provide even greater application for this technology.

In addition to magnetic targeting, physiochemical properties of IONPs such as surface charge are of great importance to application of MFECD. One reason for this may be the greater cellular uptake profile of the positively charged AmS-IONPs. As reported previously, the negatively charged EDT-IONPs have a lower cellular uptake compared with positively charged AmS-IONPs. Furthermore, the differences in EDT-IONP and AmS-IONP accumulation profiles in the bEnd.3 cells were unaffected by hyperosmotic conditions, suggesting endocytotic activity was not altered by hyperosmotic conditions. These findings, together with the increased permeability of EDT-IONPs in hyperosmotic conditions, suggest the negatively charged IONPs are more likely to favor the paracellular route, and thus are more suitable for applications with MFECD.

An additional consideration for the differences in permeability of the IONPs is the influence of surface charge on colloidal stability. The stability of a colloidal suspension is estimated by the magnitude of the surface (zeta) potential

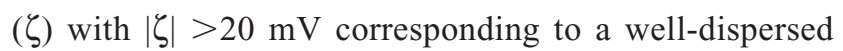
suspension. ${ }^{41}$ NP surface charge is affected strongly by the properties of the suspension medium (eg, $\mathrm{pH}$, ionic strength, presence of plasma protein). At physiological $\mathrm{pH}$, there is a slight excess of hydroxide ions in solution. The positive surface charge of the AmS-IONPs attracts electrostatically a fraction of the excess hydroxide ions, effectively lowering the zeta potential by means of charge cancellation. It is possible that at physiological $\mathrm{pH}$, there are sufficient counter-ions in solution to reduce the surface charge of the AmS-IONPs below the point of stability, leading to aggregation. This is seen clearly in the PCS results collected for AmS- and EDTIONPs. As demonstrated in previous studies, the aggregation observed with the AmS-IONP may be further enhanced through interactions (eg, electrostatic and friction) between NPs and the various proteins present in the cell culture media. ${ }^{42-45}$ The combination of these effects leads ultimately to the reduced permeability of the AmS-IONPs across the blank PC membrane when compared with the EDT-IONPs. Aggregation of the positively charged AmS-IONPs, along with their high uptake in endothelial cell monolayers making up the BBB, suggest that IONPs with negative surface charge benefit most greatly from MFECD. Our results suggest that to maximize the effectiveness of MFECD on NP permeability, great care must be exercised in the design of NP coating to ensure long-term colloidal stability at physiological $\mathrm{pH}$.

The use of MFECD together with transient disruption of BBB permeability as a method for enhancing IONP delivery to the brain represents a substantial departure from vesicular transport pathways commonly examined. Multifunctional NPs have been developed by grafting BBB targeting moieties, such as Angiopep-2 targeting low-density lipoprotein receptor-related protein receptor and transferin antibodies targeting transferrin receptor, to improve the BBB penetration via receptor-mediated transcytosis. ${ }^{46,47}$ In addition to active targeting, adsorptive mediated transcytosis triggered by electrostatic interactions between the positively charged moiety on the NPs and negatively charged cell membrane of nanoparticles has also been evaluated for BBB delivery. ${ }^{48}$ Although NP delivery to the brain via receptor-mediated and adsorptive transcytosis has been reported, there are significant hurdles that remain. For example, the physiochemical properties of the particles can be altered by the covalent attachment of ligands resulting in reduced circulation times, which offset the potential benefits of active targeting. ${ }^{49}$ Most important, the delivery efficiency via transcytosis as a percentage of the injected dose is rather limited: Only $0.25 \%$ of an injected dose of Angiopep-conjugated polyamidoamine dendrimer NPs per gram of brain tissue 2 hours after tail vein injection in Balb/c mice has been reported..$^{50}$ Even lower brain delivery efficiency was obtained using NPs coated with a 29-amino-acid peptide derived from the rabies virus glycoprotein 4 hours after intravenous injection in mice. ${ }^{51}$ In addition, several in vivo studies have reported low efficiencies for brain delivery, using the transcytosis route. ${ }^{52,53}$ Examination of transcytosis in cell culture models of the BBB show relatively low permeability with prion-labeled NPs (ie, $6 \%$ flux) and cationic polyethylenimine-coated NPs (ie, 3.4\% flux) after 18-hour permeability studies. ${ }^{54}$ These permeability rates are consistent with the low permeability observed for the positively charged AmS-IONPs in the present study.

\section{Conclusion}

This proof-of-concept study is concerned with the feasibility of using MFECD to enhance the permeability of charged IONPs for potential brain-targeted drug delivery. These studies show that MFECD, when paired with disruption of tight junctions, can significantly enhance IONP permeability in an in vitro model of the BBB. The effects of MFECD were most apparent with the negatively charged EDT-IONPs, 
consistent with enhanced bulk flow permeability of IONPs across transiently disrupted brain microvessel endothelial cells. The MFECD of EDT-IONPs provided higher delivery efficiency compared with either passive or active targeting of BBB vesicular transport processes. The use of MFECD in combination with methods for transient disruption of $\mathrm{BBB}$ permeability represents a potential method for enhancing drug delivery to the brain.

\section{Acknowledgments}

This study was funded by research grants from the Dr HT Thorlakson Foundation (DWM) and the Collaborative Health Research Program sponsored by the Canadian Institutes of Health Research and Natural Science and Engineering Research Council of Canada (DWM, JvL). This work was also financially supported by the Ohio Third Frontier Ohio Research Scholar Program Research Cluster on Surfaces in Advanced Materials (TH), which also supports the cryotransmission electron microscopy facility at the Liquid Crystal Institute, Kent State University, where current transmission electron microscopy data were obtained. Graduate student fellowship support was provided by the Natural Science and Engineering Research Council of Canada (ZS) and the University of Manitoba (VY, YW).

\section{Disclosure}

The authors report no conflicts of interest in this work.

\section{References}

1. Pardridge WM. Why is the global CNS pharmaceutical market so under-penetrated? Drug Discov Today. 2002;7(1):5-7.

2. Daneman R. The blood-brain barrier in health and disease. Ann Neurol. 2012;72(5):648-672.

3. On NH, Chen F, Hinton M, Miller DW. Assessment of P-glycoprotein activity in the Blood-Brain Barrier (BBB) using Near Infrared Fluorescence (NIRF) imaging techniques. Pharm Res. 2011;28(10): 2505-2515.

4. Nicolazzo JA, Katneni K. Drug transport across the blood-brain barrier and the impact of breast cancer resistance protein (ABCG2). Curr Top Med Chem. 2009;9(2):130-147.

5. Löscher W, Potschka H. Blood-brain barrier active efflux transporters: ATP-binding cassette gene family. Neuro Rx. 2005;2(1):86-98.

6. Zhang Y, Schuetz JD, Elmquist WF, Miller DW. Plasma membrane localization of multidrug resistance-associated protein homologs in brain capillary endothelial cells. J Pharmacol Exp Ther. 2004;311(2): 449-455.

7. Pardridge WM. Drug targeting to the brain. Pharm Res. 2007;24(9): 1733-1744.

8. Tietze R, Lyer S, Dürr S, Alexiou C. Nanoparticles for cancer therapy using magnetic forces. Nanomedicine (Lond). 2012;7(3):447-457.

9. Zhu L, Wang D, Wei X, et al. Multifunctional $\mathrm{pH}$-sensitive superparamagnetic iron-oxide nanocomposites for targeted drug delivery and MR imaging. J Control Release. 2013;169(3):228-238.

10. Fan CH, Ting CY, Lin HJ, et al. SPIO-conjugated, doxorubicin-loaded microbubbles for concurrent MRI and focused-ultrasound enhanced brain-tumor drug delivery. Biomaterials. 2013;34(14):3706-3715.
11. Lee GY, Qian WP, Wang L, et al. Theranostic nanoparticles with controlled release of gemcitabine for targeted therapy and MRI of pancreatic cancer. ACS Nano. 2013;7(3):2078-2089.

12. Xu C, Sun S. New forms of superparamagnetic nanoparticles for biomedical applications. Adv Drug Deliv Rev. 2013;65(5):732-743.

13. Kenzaoui BH, Bernasconi CC, Hofmann H, Juillerat-Jeanneret L. Evaluation of uptake and transport of ultrasmall superparamagnetic iron oxide nanoparticles by human brain-derived endothelial cells. Nanomedicine (Lond). 2012;7(1):39-53.

14. Lee MJ, Veiseh O, Bhattarai N, et al. Rapid pharmacokinetic and biodistribution studies using cholorotoxin-conjugated iron oxide nanoparticles: a novel non-radioactive method. PLoS One. 2010;5(3):e9536.

15. Peer D, Karp JM, Hong S, Farokhzad OC, Margalit R, Langer R. Nanocarriers as an emerging platform for cancer therapy. Nat Nanotechnol. 2007;2(12):751-760.

16. Alexiou C, Schmid RJ, Jurgons R, et al. Targeting cancer cells: magnetic nanoparticles as drug carriers. Eur Biophys J. 2006;35(5):446-450.

17. On NH, Mitchell R, Savant SD, Bachmeier CJ, Hatch GM, Miller DW. Examination of blood-brain barrier (BBB) integrity in a mouse brain tumor model. J Neurooncol. 2013;111(2):133-143.

18. Sun Z, Yathindranath V, Worden M, et al. Characterization of cellular uptake and toxicity of aminosilane-coated iron oxide nanoparticles with different charges in central nervous system-relevant cell culture models. Int J Nanomedicine. 2013;8:961-970.

19. Yathindranath V, Rebbouh L, Moore DF, Miller DW, van Lierop J, Hegmann T. A versatile method for the reductive, one-pot synthesis of bare, hydrophilic and hydrophobic magnetite nanoparticles. Adv Funct Mater. 2011;21(8):1457-1464.

20. Yathindranath V, Sun Z, Worden M, et al. One-pot synthesis of iron oxide nanoparticles with functional silane shells: a versatile general precursor for conjugations and biomedical applications. Langmuir. 2013;29(34):10850-10858.

21. Hoff D, Sheikh L, Bhattacharya S, Nayar S, Webster TJ. Comparison study of ferrofluid and powder iron oxide nanoparticle permeability across the blood-brain barrier. Int J Nanomedicine. 2013;8:703-710.

22. Li G, Simon MJ, Cancel LM, et al. Permeability of endothelial and astrocyte cocultures: in vitro blood-brain barrier models for drug delivery studies. Ann Biomed Eng. 2010;38(8):2499-2511.

23. Berne BJ, Pecora R. Dynamic Light Scattering: With Applications to Chemistry, Biology, and Physics. New York: Dover Publications; 2000.

24. Wang J, Chen Y, Chen B, et al. Pharmacokinetic parameters and tissue distribution of magnetic $\mathrm{Fe}(3) \mathrm{O}(4)$ nanoparticles in mice. Int $J$ Nanomedicine. 2010;5:861-866.

25. Petri B, Bootz A, Khalansky A, et al. Chemotherapy of brain tumour using doxorubicin bound to surfactant-coated poly(butyl cyanoacrylate) nanoparticles: revisiting the role of surfactants. J Control Release. 2007; 117(1):51-58.

26. Qiao R, Jia Q, Hüwel S, et al. Receptor-mediated delivery of magnetic nanoparticles across the blood-brain barrier. ACS Nano. 2012;6(4): 3304-3310.

27. Etame AB, Smith CA, Chan WC, Rutka JT. Design and potential application of PEGylated gold nanoparticles with size-dependent permeation through brain microvasculature. Nanomedicine. 2011;7(6):992-1000.

28. Boockvar JA, Tsiouris AJ, Hofstetter CP, et al. Safety and maximum tolerated dose of superselective intraarterial cerebral infusion of bevacizumab after osmotic blood-brain barrier disruption for recurrent malignant glioma. Clinical article. J Neurosurg. 2011;114(3):624-632.

29. Kiptoo P, Sinaga E, Calcagno AM, et al. Enhancement of drug absorption through the blood-brain barrier and inhibition of intercellular tight junction resealing by E-cadherin peptides. Mol Pharm. 2011;8(1): 239-249.

30. On NH, Savant S, Toews M, Miller DW. Rapid and reversible enhancement of blood-brain barrier permeability using lysophosphatidic acid. J Cereb Blood Flow Metab. 2013;33(12):1944-1954.

31. Borlongan CV, Glover LE, Sanberg PR, Hess DC. Permeating the blood brain barrier and abrogating the inflammation in stroke: implications for stroke therapy. Curr Pharm Des. 2012;18(25):3670-3676. 
32. Hynynen K. Ultrasound for drug and gene delivery to the brain. $A d v$ Drug Deliv Rev. 2008;60(10):1209-1217.

33. Muldoon LL, Nilaver G, Kroll RA, et al. Comparison of intracerebral inoculation and osmotic blood-brain barrier disruption for delivery of adenovirus, herpesvirus, and iron oxide particles to normal rat brain. Am J Pathol. 1995;147(6):1840-1851.

34. Liu HL, Hua MY, Yang HW, et al. Magnetic resonance monitoring of focused ultrasound/magnetic nanoparticle targeting delivery of therapeutic agents to the brain. Proc Natl Acad Sci U S A. 2010;107(34): $15205-15210$.

35. Burgess A, Hynynen K. Noninvasive and targeted drug delivery to the brain using focused ultrasound. ACS Chem Neurosci. 2013;4(4): 519-526.

36. Carenza E, Barceló V, Morancho A, et al. In vitro angiogenic performance and in vivo brain targeting of magnetized endothelial progenitor cells for neurorepair therapies. Nanomedicine. 2014;10(1):225-234.

37. Kong SD, Lee J, Ramachandran S, et al. Magnetic targeting of nanoparticles across the intact blood-brain barrier. J Control Release. 2012;164(1):49-57.

38. Chertok B, David AE, Yang VC. Brain tumor targeting of magnetic nanoparticles for potential drug delivery: effect of administration route and magnetic field topography. J Control Release. 2011;155(3):393-399.

39. Chertok B, Moffat BA, David AE, et al. Iron oxide nanoparticles as a drug delivery vehicle for MRI monitored magnetic targeting of brain tumors. Biomaterials. 2008;29(4):487-496.

40. Zhang J, Shin MC, Yang VC. Magnetic targeting of novel heparinized iron oxide nanoparticles evaluated in a 9L-glioma mouse model. Pharm Res. 2014;31(3):579-592.

41. Delgado AV, González-Caballero F, Hunter RJ, Koopal LK, Lyklema J; International Union of Pure and Applied Chemistry, Physical and Biophysical Chemistry Division IUPAC Technical Report. Measurement and interpretation of electrokinetic phenomena. J Colloid Interface Sci. 2007;309(2):194-224.

42. Lesniak A, Salvati A, Santos-Martinez MJ, Radomski MW, Dawson KA, Åberg C. Nanoparticle adhesion to the cell membrane and its effect on nanoparticle uptake efficiency. J Am Chem Soc. 2013; 135(4):1438-1444.

43. Jedlovszky-Hajdú A, Bombelli FB, Monopoli MP, Tombácz E, Dawson KA. Surface coatings shape the protein corona of SPIONs with relevance to their application in vivo. Langmuir. 2012;28(42): 14983-14991.
44. Ruh H, Kühl B, Brenner-Weiss G, Hopf C, Diabaté S, Weiss C. Identification of serum proteins bound to industrial nanomaterials. Toxicol Lett. 2012;208(1):41-50.

45. Safi M, Courtois J, Seigneuret M, Conjeaud H, Berret JF. The effects of aggregation and protein corona on the cellular internalization of iron oxide nanoparticles. Biomaterials. 2011;32(35):9353-9363.

46. Xin H, Sha X, Jiang X, Zhang W, Chen L, Fang X. Anti-glioblastoma efficacy and safety of paclitaxel-loading Angiopep-conjugated dual targeting PEG-PCL nanoparticles. Biomaterials. 2012;33(32): 8167-8176.

47. Yemişci M, Gürsoy-Özdemir Y, Caban S, Bodur E, Capan Y, Dalkara T. Transport of a caspase inhibitor across the blood-brain barrier by chitosan nanoparticles. Methods Enzymol. 2012;508:253-269.

48. Lu W, Wan J, She Z, Jiang X. Brain delivery property and accelerated blood clearance of cationic albumin conjugated pegylated nanoparticle. J Control Release. 2007;118(1):38-53.

49. McNeeley KM, Karathanasis E, Annapragada AV, Bellamkonda RV. Masking and triggered unmasking of targeting ligands on nanocarriers to improve drug delivery to brain tumors. Biomaterials. 2009; 30(23-24):3986-3995.

50. Ke W, Shao K, Huang R, et al. Gene delivery targeted to the brain using an Angiopep-conjugated polyethyleneglycol-modified polyamidoamine dendrimer. Biomaterials. 2009;30(36):6976-6985.

51. Chen W, Zhan C, Gu B, et al. Targeted brain delivery of itraconazole via RVG29 anchored nanoparticles. J Drug Target. 2011;19(3): 228-234.

52. van Rooy I, Mastrobattista E, Storm G, Hennink WE, Schiffelers RM. Comparison of five different targeting ligands to enhance accumulation of liposomes into the brain. J Control Release. 2011;150(1):30-36.

53. Xie Y, Ye L, Zhang X, et al. Transport of nerve growth factor encapsulated into liposomes across the blood-brain barrier: in vitro and in vivo studies. J Control Release. 2005;105(1-2):106-119.

54. Georgieva JV, Kalicharan D, Couraud PO, et al. Surface characteristics of nanoparticles determine their intracellular fate in and processing by human blood-brain barrier endothelial cells in vitro. Mol Ther. 2011;19(2):318-325. 


\section{Supplementary material}

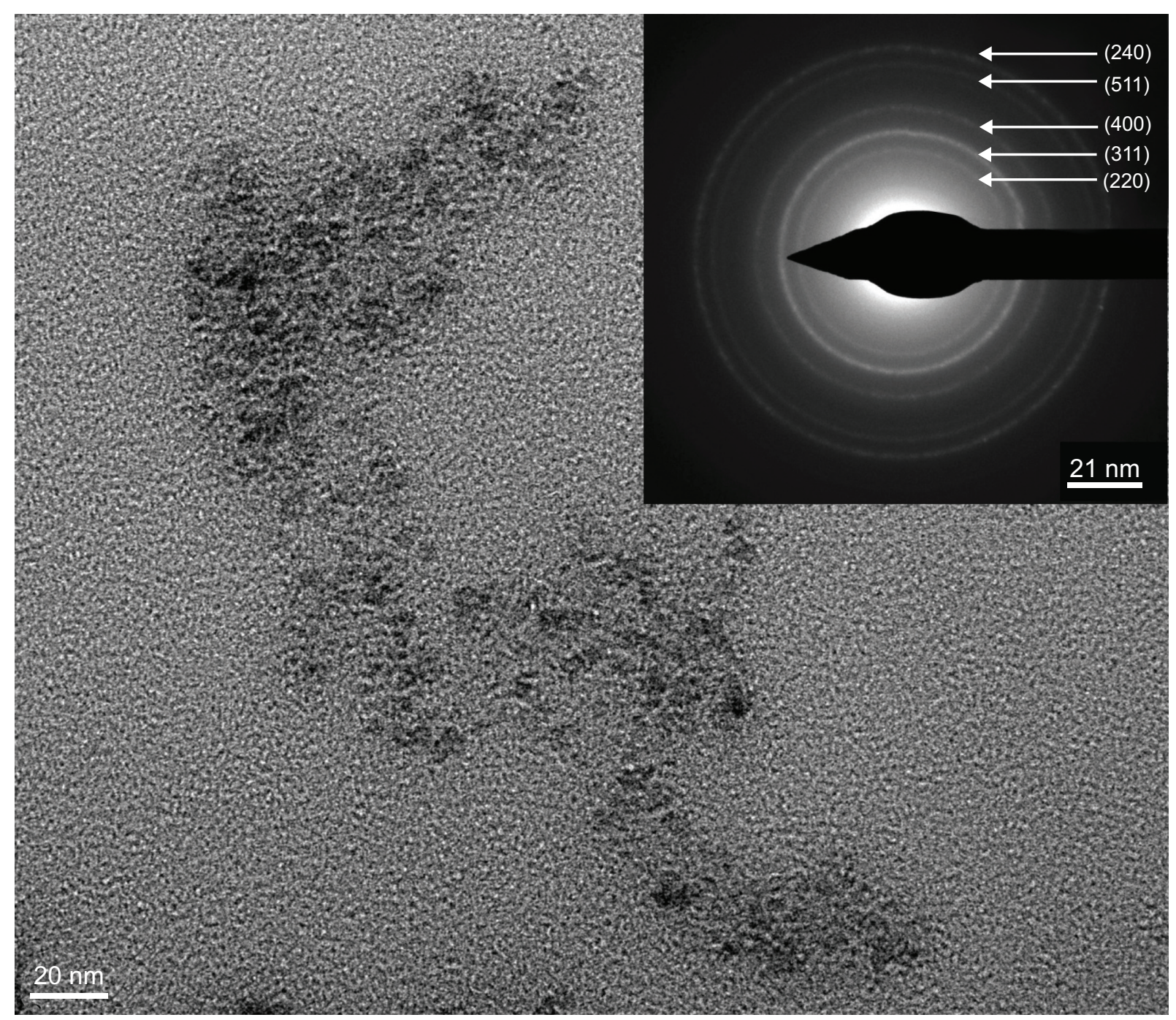

Figure SI Transmission electron microscopy image of $\mathrm{N}$-(trimethoxysilylpropyl)ethylenediaminetriacetate-iron oxide nanoparticles. Inset shows selected area electron diffraction pattern of these particles.

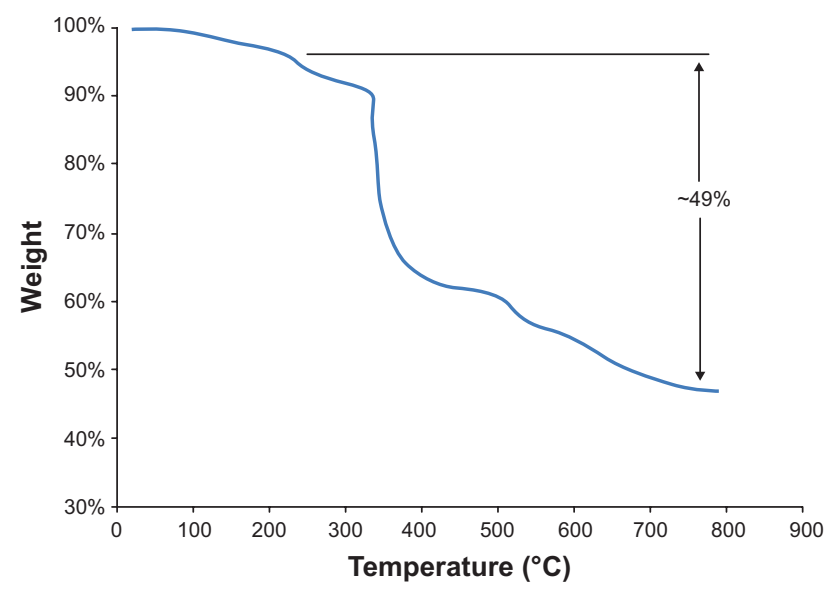

Figure S2 Thermal gravimetric analysis plot for N-(trimethoxysilylpropyl)ethylene diaminetriacetate-iron oxide nanoparticles showing approximately $49 \%$ weight loss after desorption of water.

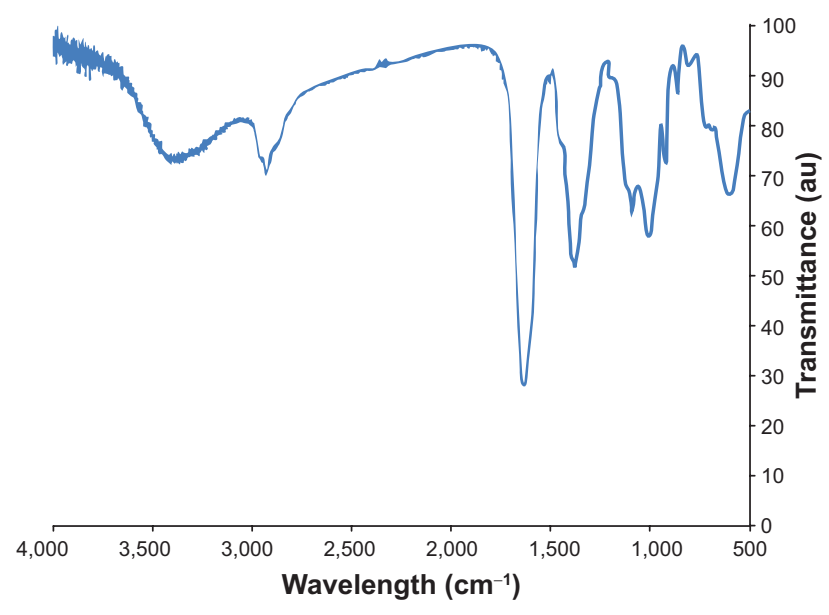

Figure S3 Fourier transform infrared spectroscopy spectrum of $\mathrm{N}$-(trimethoxysilylpropyl)ethylenediaminetriacetate-iron oxide nanoparticles. 


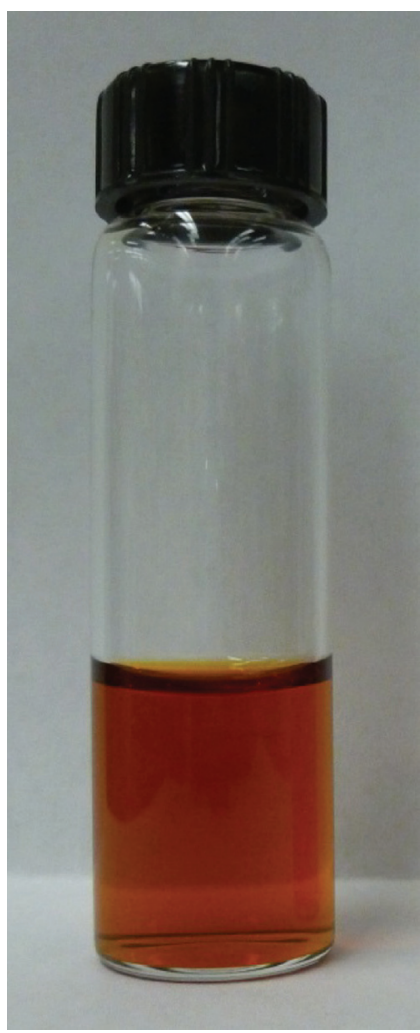

Figure S4 Picture of a dispersion of $\mathrm{N}$-(trimethoxysilylpropyl)ethylenediaminetriacetate-iron oxide nanoparticles in water.

\section{Nanoparticle characterization}

Successful coating of the iron oxide nanoparticles with $\mathrm{N}$-(trimethoxysilylpropyl)ethylenediaminetriacetate (EDT)silane was demonstrated first with Fourier transform infrared spectroscopy, using a Nicolet Magna-IR Spectrometer 550 (Thermo Fisher Scientific, Waltham, MA, USA). Sample preparation involved pelletizing the particles in $\mathrm{KBr}$. Characteristic peaks at $\sim 2,950 \mathrm{~cm}^{-1}$ indicate $\mathrm{C}-\mathrm{H}$ stretching. The strong peak between $\sim 1,550 \mathrm{~cm}^{-1}$ to $\sim 1,630 \mathrm{~cm}^{-1}$ is associated with asymmetric $\mathrm{COO}^{-}$stretching. The collection of peaks between 1,350-1,450 $\mathrm{cm}^{-1}$ are associated with symmetrical $\mathrm{COO}^{-}$stretching and $\mathrm{CH}_{2}-\mathrm{COO}^{-}$scissoring. Finally, the peaks at $\sim 990 \mathrm{~cm}^{-1}$ and $\sim 1,100 \mathrm{~cm}^{-1}$ can be attributed to Si-O-Si and Si-O-R vibrations.

An estimate of the amount of EDT-silane on the surface of the particles was obtained with a TA Instruments (New Castle, DE, USA) Hi-Res TGA 2950. Figure S2 shows the results. Initial weight loss is a result of water desorption, after which the EDT-silane coating begins to burn off at approximately $200^{\circ} \mathrm{C}$ until the weight loss stabilizes before $800^{\circ} \mathrm{C}$. Total weight loss after water desorption is approximately $49 \%$.
International Journal of Nanomedicine

\section{Publish your work in this journal}

The International Journal of Nanomedicine is an international, peerreviewed journal focusing on the application of nanotechnology in diagnostics, therapeutics, and drug delivery systems throughout the biomedical field. This journal is indexed on PubMed Central, MedLine, CAS, SciSearch ${ }^{\circledR}$, Current Contents ${ }^{\circledR} /$ Clinical Medicine,

\section{Dovepress}

Journal Citation Reports/Science Edition, EMBase, Scopus and the Elsevier Bibliographic databases. The manuscript management system is completely online and includes a very quick and fair peer-review system, which is all easy to use. Visit http://www.dovepress.com/ testimonials.php to read real quotes from published authors. 